\title{
Length Bidisperse Carbon Nanotubes Dispersions in Thermotropic Liquid Crystals
}

\author{
V. Popa-Nita ${ }^{1}$ and S. Buček ${ }^{2}$ \\ ${ }^{1}$ Faculty of Physics, University of Bucharest, P.O. Box MG-11, 077125 Bucharest, Romania \\ ${ }^{2}$ Osnovna šola I Murska Sobota, Štefana Kovača 32, 9000 Murska Sobota, Slovenia \\ Correspondence should be addressed to V. Popa-Nita, v.popanita@gmail.com
}

Received 9 May 2012; Accepted 27 July 2012

Academic Editor: Ali Hussain Reshak

Copyright ( 2012 V. Popa-Nita and S. Buček. This is an open access article distributed under the Creative Commons Attribution License, which permits unrestricted use, distribution, and reproduction in any medium, provided the original work is properly cited.

\begin{abstract}
We study nematic liquid crystal driven alignment of carbon nanotubes dispersed in them. We extend the mesoscopic model presented in (P. Van der Schoot et al. 2008, V. Popa-Nita, and S. Kralj 2010) including the effect of length bidispersity of carbon nanotubes. The free energy of the mixture is written as the sum of the Doi free energy for lyotropic nematic ordering of the two carbon nanotubes types, the Landau-de Gennes free energy for the thermotropic ordering of liquid crystal, and the coupling term between liquid crystal molecules and carbon nanotubes. The phase ordering of the mixtures is analyzed as a function of volume fraction, the strength of coupling, and the temperature.
\end{abstract}

\section{Introduction}

Carbon nanotubes (CNTs) either consist of a single sheet of carbon rolled up into a cylinder with a diameter of about one nanometer (single-walled nanotubes-SWNTs), or are built up of multiple carbon sheets producing rods with diameters ranging from a few to tens of even hundreds of nanometer (multiwalled nanotubes-MWNTs). These nanoparticles have a large aspect ratio which varies from hundred to many thousands. CNTs exhibit most of their remarkable properties in a single direction, that is, along the tube axis [1].

Most of CNTs extraordinary properties of potential use in various applications could be realized in relatively wellaligned samples. Recently, it has been shown that liquid crystal alignment could trigger spontaneous ordering of CNTs with remarkably high degree of ordering [2-5]. CNTs orient parallel to average direction of liquid crystal (LC) alignment with an orientational order parameter between 0.6 to 0.9 [69]. Both, thermotropic $[6,7,9]$ and lyotropic nematic LC phases [8] have been successfully applied as aligning solvents.

Theoretically, equilibrium orientation of a single elongated particle immersed in a nematic LC phase in rather well explored [10-17]. Continuum theory predicts alignment of the particle's longer axis along the nematic director $\vec{n}$ for different types of boundary conditions in the strong anchoring limit [10-12]. In the weak anchoring limit, a rodlike particle may orient either along or perpendicular to the nematic director depending on the boundary conditions [11].

The theoretical study of the collective behaviour of anisotropic nanoparticles dispersed in isotropic solvents or in liquid crystals is based on the observation that they can be considered essentially as rigid-rod polymers with a large aspect ratio [18]. The Onsager's theory for the electrostatic repulsion of long rigid rods has been used to investigate the phase behaviour of SWNTs dispersed into organic and aqueous solvents [19]. In an adequate solubility, when the van der Waals attractive interaction between CNTs is overcome by strong repulsive interrods potential, the ordered phase of CNTs can form at room temperature. On the contrary, the van der Waals attractive interactions between the rods are strong, and as a result, only extremely dilute solutions of SWNTs are thermodynamically stable and no liquid crystal phases form at room temperature. The liquid crystalline of CNTs with and without van der Waals interactions has been analyzed by using the density functional theory [20] In the presence of van der Waals interaction, the nematic 
as well as the columnar phases occur in the temperaturepacking fraction phase diagram in a wide range of very high temperatures. In the absence of van der Waals interaction, with an increase of packing fraction, the system undergoes an isotropic-nematic phase transition via a biphasic region. The isotropic-nematic packing fraction decreases with the increase of the aspect ratio of CNTs. To describe the dispersion of SWNTs in superacids, the Onsager theory for rigid rods was extended to include the length polydispersity and solvent-mediated attraction and repulsion [21]. The main conclusion of these theoretical models is that to obtain liquid crystal phases of CNTs at room temperature the strong van der Waals interaction between them must be screened out. This requires an adequate solubility of the CNTs down to the level of individual tube. Note that nanoparticles could strongly interact via LC ordering [22$25]$ and also topological defects $[24,26]$ as recent studies reveal. Furthermore, the CNTs could induce into system some degree of quenched disorder [27].

In $[28,29]$, a phenomenological theory for predicting the alignment of length monodisperse CNTs dispersions in thermotropic nematic liquid crystals was developed. The Landau-de Gennes free energy for thermotropic ordering of the liquid crystal solvent was combined with the Doi free energy for the lyotropic nematic ordering of CNTs caused by excluded-volume interactions between them. In the first paper [28], the interaction between CNTs and liquid crystal molecules is thought to be sufficiently weak to not cause any director field deformations in the nematic host fluid. In the second paper [29], the strong anchoring limit case was analyzed for which the nematic ordering around nanotubes is apparently distorted and consequently relatively strong long range and anisotropic interactions can emerge within the system. In both cases, the phase ordering of the binary mixture as a function of volume fraction of CNTs, the strength of the coupling and the temperature were analyzed.

Usually, after the acid treatment and ultrasonification (to enhance the dispersibility and stability of the CNTs suspensions), depending on the temperature water bath and time of ultrasonification, the obtained CNTs have different lengths and diameters [7, 30]. The influence of length bidispersity on the phase diagram and alignment of CNTs is the subjects of the present paper. As the first step we extended our mesoscopic model $[28,29]$ and consider length bidispersity of CNTs.

The remainder of this paper is organised as follows. In Section 2, we introduce the free energy of mixture of CNTs and LC. In Section 3, we present our results and in Section 4 we draw conclusions.

\section{Model}

The mixture is characterized by the volume fractions of the three components:

$$
\Phi_{i}=\frac{N_{i} v_{i}}{\sum_{j=1}^{3} N_{j} v_{j}} \quad \text { with } \sum_{i=1}^{3} \Phi_{i}=1,
$$

where $N_{i}$ is the number of molecules of component $i$ ( $i=1$ defines the liquid crystal, $i=2$ the CNTs of length $L_{02}$ and diameter $D$, and $i=3$ the length $L_{03}$ and diameter $D$ ) and $v_{i}$ is the volume of a particle of component $i$. For latter convenience, we introduce scaled CNT lengths $L_{j}=L_{0 j} / D$ $(j=2,3)$.

The degree of alignment of every component of the mixture is characterized by the scalar order parameter $S_{i}$ [31]. The corresponding isotropic liquid of the component $i$ is characterized by $S_{i}=0$ while a perfectly oriented nematic phase would correspond to $S_{i}=1$.

2.1. Free Energy. The free energy per unit volume of mixture is expressed as

$$
f=f_{\mathrm{CNT}}+f_{\mathrm{LC}}+f_{c},
$$

where $f_{\mathrm{CNT}}$ describes contribution of the two CNT components dispersed in the LC fluid, $f_{\mathrm{LC}}$ represents the free energy density of nematic liquid crystal order, while $f_{c}$ takes into account the coupling between LC molecules and CNTs, respectively.

The free energy density of CNTs is given by $[28,29]$

$$
\begin{aligned}
f_{\mathrm{CNT}}= & k_{B} T \sum_{i=2}^{3}\left\{\frac{\Phi_{i}}{v_{i}} \ln \Phi_{i}\right. \\
& \left.+\frac{L_{i} \Phi_{i}^{2}}{6 v_{i}}\left[\left(\frac{3}{L_{i} \Phi_{i}}-1\right) S_{i}^{2}-\frac{2}{3} S_{i}^{3}+S_{i}^{4}\right]\right\} \\
& -\gamma_{23} \Phi_{2} \Phi_{3} S_{2} S_{3},
\end{aligned}
$$

where $k_{B}$ is the Boltzmann constant and $T$ is the temperature. The first term in the sum represents the entropic isotropic contribution due to mixing of the two CNT components neglecting their orientational degree of ordering [32]. In the limit of small volume fractions of the two CNTs components, the isotropic (Flory-Huggins) interaction terms of the form $\chi \Phi_{i} \Phi_{j}$ can be neglected. The second term in the sum (usually called Doi free energy) that accounts for the gain in free volume and the loss of orientational entropy upon ordering of the rods for every CNT component is obtained starting from the Onsager theory [33] and using the Smoluchovsky equation. The details are presented in [34-36]. We mention that the Doi model neglects the van der Waals attractions between CNTs which are responsible for their tendency to form bundles. Minimizing the Doi free energy, a first-order isotropic-nematic phase transition is obtained which takes place at $\Phi_{i}^{(\mathrm{NI})}=2.7 / L_{i}$ and $S_{i}^{(\mathrm{NI})}=1 / 3$. The last term in (3) represents the interaction energy between the different CNTs types, where the interaction parameter $\gamma_{23}$ is given by

$$
\gamma_{23} \sim \frac{8 k_{B} T}{\pi D^{3}}
$$

This result is obtained starting with the Onsager theory for the binary mixture and using the Smolukovsky equation [37]. The second term in (2) is the Landau-de Gennes free energy density [31] which describes the weakly first-order nematic-isotropic phase transition of thermotropic LC:

$$
f_{\mathrm{LC}}=\left(1-\Phi_{2}-\Phi_{3}\right)\left[a\left(T-T^{*}\right) S_{1}^{2}-B S_{1}^{3}+C S_{1}^{4}\right]
$$


where $\left(1-\Phi_{2}-\Phi_{3}\right)$ accounts for the part of the volume not taken up by LC. The quantities $T^{*}, a, B$, and $C$ are material-dependent constants, where $T^{*}$ denotes the spinodal temperatures limit of the isotropic phase of the pure LC. This free energy describes a weakly first-order nematicisotropic phase transition. At $T=T_{\mathrm{NI}}=T^{*}+B^{2} /(4 a C)$, the two phases, nematic $\left(S_{1}^{(\mathrm{NI})}=B /(2 C)\right)$ and isotropic $\left(S_{1}=0\right)$ coexist in equilibrium.

The third term in (2) represents the coupling between the liquid crystal molecules and the two CNT species. The resulting coupling term structure in both anchoring limits (weak and strong) we estimated in $[28,29]$. The two limits are defined by the ratio $D / d_{e}[10-12]$, where $d_{e}=K / W$ stands for the surface extrapolation, $W$ is the anchoring energy of an LC-nanotube interface, $K$ is the average Frank nematic elastic constant [31]. For typical thermotropic LCs and nanotubes, it holds $\approx 10^{-11} \mathrm{~N}, W \approx 10^{-6} \mathrm{~N} / \mathrm{m}[38-$ 41 ], and the penetration length should be vastly in excess of $\mu \mathrm{m}$. The radius of CNTs is at the $\mathrm{nm}$ scale, therefore, $D \ll d_{e}$. Consequently, only the weak-anchoring limit needs to be considered, as already concluded by Lynch and Patrick [6]. The corresponding free energy density of coupling is approximately given by [28]

$$
\begin{aligned}
f_{c}= & -\gamma_{12}\left(1-\Phi_{2}-\Phi_{3}\right) \Phi_{2} S_{1} S_{2} \\
& -\gamma_{13}\left(1-\Phi_{2}-\Phi_{3}\right) \Phi_{3} S_{1} S_{3} .
\end{aligned}
$$

The coupling parameters $\gamma_{12}$ and $\gamma_{13}$ depend only on the anchoring energy of CNTs at the LC molecules surface and the diameter of CNTs [28] (i.e., it does not depend on the length of CNT). Within this approximation, it holds

$$
\gamma_{12} \sim \gamma_{13}=\gamma_{1}=\frac{4 W}{3 D} .
$$

Therefore,

$$
f_{c}=-\gamma_{1}\left(1-\Phi_{2}-\Phi_{3}\right) S_{1}\left(\Phi_{2} S_{2}+\Phi_{3} S_{3}\right) .
$$

2.2. Material and Geometrical Parameters. In this paper, we will consider the following typical geometrical and material values. For CNTs, we set $D=2 \mathrm{~nm}, L_{02}=400 \mathrm{~nm}$, and $L_{03}=800 \mathrm{~nm}$. Considering $T=300 \mathrm{~K}$, the coupling constant $\gamma_{23}$ has the value $10^{6} \mathrm{~J} / \mathrm{m}^{3}$, which we use in the following. For representative LC material, we chose pentylcyanobiphenyl (5CB), for which $T^{*}=307.55 \mathrm{~K}, a \approx 5.2 \cdot 10^{4} \mathrm{~J} / \mathrm{m}^{3} \mathrm{~K}, B \approx$ $5.3 \cdot 10^{5} \mathrm{~J} / \mathrm{m}^{3}, C \approx 9.7 \cdot 10^{5} \mathrm{~J} / \mathrm{m}^{3}$, and $K \sim 10^{-11} \mathrm{~N}[38-41]$. This chose yields $S_{1}^{(\mathrm{NI})}=0.275, T_{\mathrm{NI}}=308.95 \mathrm{~K}$. For the LCCNT interaction, we use $\gamma_{1}=4 W / 3 D \approx 10^{3} \mathrm{~N} / \mathrm{m}^{2}$, where we have assumed $W \sim 10^{-6} \mathrm{~N} / \mathrm{m}$ and $D=2 \mathrm{~nm}$.

2.3. Basic Equation. The equilibrium values of the order parameters are obtained by minimization of the free energy density ((2), (3), (5), and (6)) with respect to $S_{1}, S_{2}$, and $S_{3}$, respectively. From the corresponding equation, we find the equilibrium values of the order parameters in the nematic phase $\left(S_{i n}\right)$ and paranematic phase $\left(S_{i p}\right)$, respectively. Once the minimization procedure has been solved, the volume fractions of the coexisting (paranematic and nematic) phases are found by solving the equilibrium conditions:

$$
\begin{gathered}
\mu_{2}\left(S_{1 n}, S_{2 n}, S_{3 n}, \Phi_{2 n}, \Phi_{3 n}\right)=\mu_{2}\left(S_{1 p}, S_{2 p}, S_{3 p}, \Phi_{2 p}, \Phi_{3 p}\right), \\
\mu_{3}\left(S_{1 n}, S_{2 n}, S_{3 n}, \Phi_{2 n}, \Phi_{3 n}\right)=\mu_{3}\left(S_{1 p}, S_{2 p}, S_{3 p}, \Phi_{2 p}, \Phi_{3 p}\right), \\
g\left(S_{1 n}, S_{2 n}, S_{3 n}, \Phi_{2 n}, \Phi_{3 n}\right)=g\left(S_{1 p}, S_{2 p}, S_{3 p}, \Phi_{2 p}, \Phi_{3 p}\right),
\end{gathered}
$$

where the chemical potential of the two species of CNTs $\mu_{2}$, $\mu_{3}$, and the grand potential $g$ are defined by

$$
\mu_{2}=\frac{\partial f}{\partial \Phi_{2}} ; \quad \mu_{3}=\frac{\partial f}{\partial \Phi_{3}} ; \quad g=f-\mu_{2} \Phi_{2}-\mu_{3} \Phi_{3} .
$$

There are three equilibrium equations with four variables: $\Phi_{2 n}, \Phi_{3 n}, \Phi_{2 p}$, and $\Phi_{3 p}$. We take $\Phi_{3 p}$ as freely variable and calculate the other three $\Phi_{2 n}, \Phi_{3 n}, \Phi_{2 p}$ from the coexisting equations (9).

\section{Results}

Our aim is to analyze phase behavior of immersed CNTs in nematic LC phase as a function of $\Phi_{2}, \Phi_{3}$, and $\gamma_{1}$. The form of the coupling between CNTs and the LC molecules (8) induces two important effects: (i) the isotropic phase of CNTs transforms to a paranematic phase exhibiting relatively low degree of orientational ordering and (ii) the appearance of a critical line $\gamma_{1}^{(c)}(T)$. The reason behind this is that the coupling term $f_{c}$ is linearly proportional with $S_{i}$.

In the following, we first determine qualitatively different regimes in the $\left(T, \gamma_{1}\right)$ phase diagram on varying concentrations of CNTs. The critical line $\gamma_{1}^{(c)}(T)$ separates two qualitatively different regimes. CNTs exhibit first order transition for $\gamma_{1}<\gamma_{1}^{(c)}$. On the other hand, CNTs display gradual variations in $S_{i}$ on varying $\Phi_{i}$ if $\gamma_{1}>\gamma_{1}^{(c)}$. Afterwards, we analyze phase behavior in the $\left(\Phi_{2}, \Phi_{3}\right)$ plane for $\gamma_{1}<\gamma_{1}^{(c)}$ and $\gamma_{1}>\gamma_{1}^{(c)}$. We, henceforth, refer to the regimes $\gamma_{1}<\gamma_{1}^{(c)}$ and $\gamma_{1}>\gamma_{1}^{(c)}$ as subcritical and supercritical regime, respectively.

3.1. Phase Behaviour in the $\left(T, \gamma_{1}\right)$ Plane. The critical line $\gamma_{1}^{(c)}(T)$ is obtained by solving the equations $\partial^{2} f / \partial S_{i}^{2}=\partial^{3} f /$ $\partial S_{i}^{3}=0,(i=2,3)$. They yield at a critical point universal values for the order parameter $S_{i}^{(c)}=1 / 6$ and volume fraction $\Phi_{i}^{(c)}=18 / 7 L_{i}$. The $\gamma_{1}^{(c)}(T)$ dependence for the two species of CNTs is presented in Figure 1.

On decreasing the temperature the external field felt by the CNTs is increasing due to increasing value of $S_{1}$ and the nematic-isotropic phase transition of CNTs becomes gradual for increasingly lower values of interaction parameter $\gamma_{1}^{(c)}$. Furthermore, a critical value $\gamma_{1}$ decreases with increasing the length of CNTs. Therefore, the continuity of paranematicnematic phase transition is favoured by longer CNTs.

3.2. Phase Behaviour in the $\left(\Phi_{2}, \Phi_{3}\right)$ Plane. In what follows, we present the results in the two different cases: (i) $\gamma_{1}<\gamma_{1}^{(c)}$, 


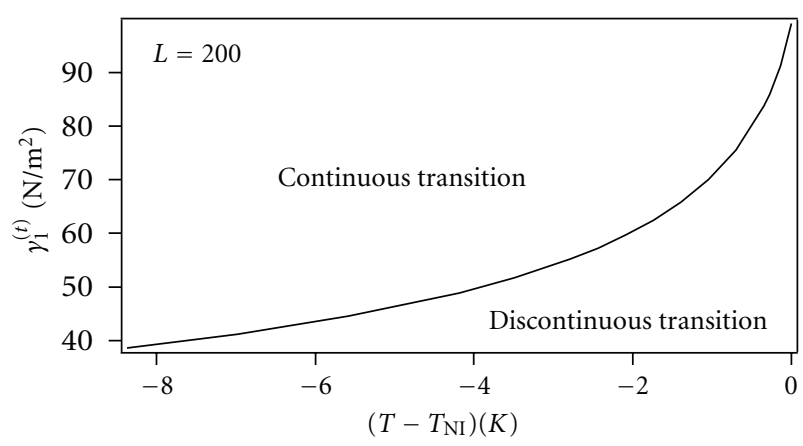

(a)

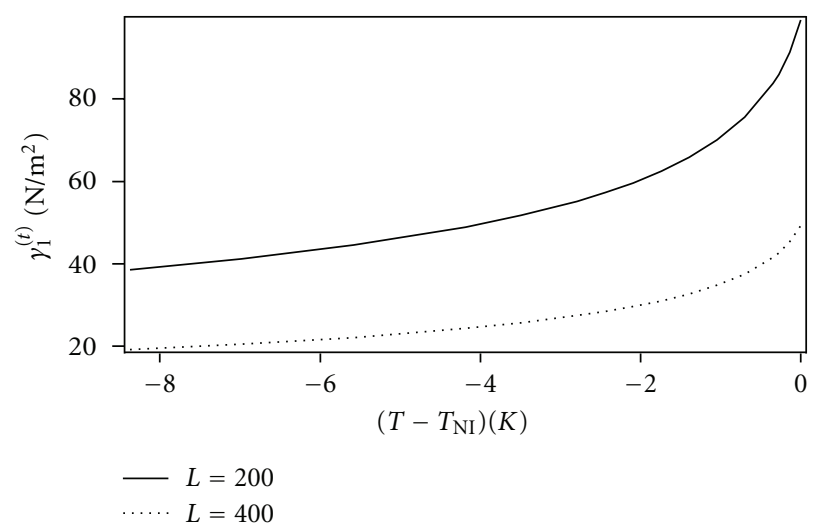

(b)

Figure 1: The critical value of the coupling parameter $\gamma_{1}^{(c)}$ as function of temperature calculated for both types of CNTs in the nematic LC phase.

when the two types of CNTs undergo first order parametricnematic phase transitions and (ii) $\gamma_{1}>\gamma_{1}^{(c)}$, where the respective transitions are replaced by gradual evolution of ordering.

3.3. Subcritical Regime. The phase diagram of the bidisperse CNTs suspension in the nematic LC for $T-T_{\mathrm{NI}}=-2.5 \mathrm{~K}$ for $\gamma_{1}=20 \mathrm{~N} / \mathrm{m}^{2}<\gamma_{1}^{(c)}$ in terms of volume fractions is shown in Figure 2.

The differences of volume fractions of both CNTs types in the nematic LC as functions of volume fraction of longer CNTs in the paranematic phase are shown in Figure 3. The temperature and the coupling parameter ( $T$ and $\gamma_{1}$ ) are the same as in Figure 2.

The figures reveal two qualitatively different regimes, to which we refer as decoupled and coupled regime, respectively. In the decoupled regime, systems exhibit monodisperse-type behavior. We label these regions by I and II in Figures 2 and 3. In the limit of very low volume fraction of the longer CNTs $\left(\Phi_{3} \rightarrow 0\right)$, the system is monodisperse containing only one types of CNTs of length $L_{02}=400 \mathrm{~nm}$ with volume fraction gap at the transition $\Delta \Phi_{2}=\Phi_{2 n}-\Phi_{2 p}=0.0143-0.0128=$ 0.0015 (subregion I). In the limit of very low volume fraction of the shorter CNTs $\left(\Phi_{2} \rightarrow 0\right)$, the system is monodisperse

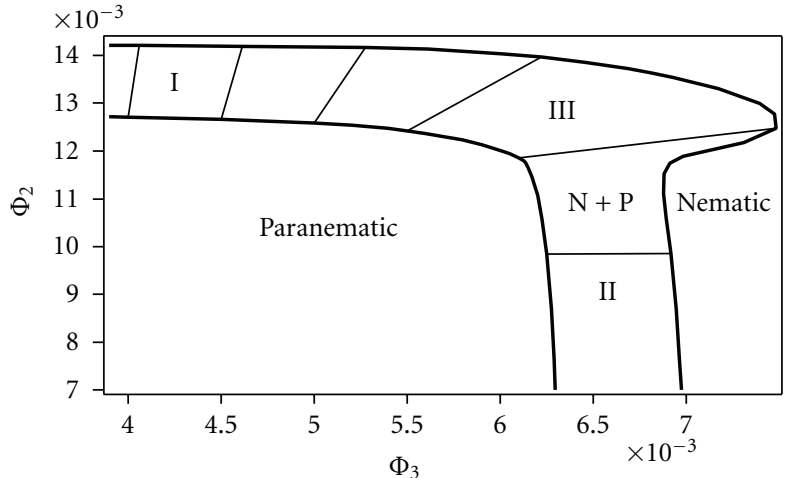

Figure 2: The $\left(\Phi_{3}, \Phi_{2}\right)$ phase diagram for a length bidisperse CNTs dispersed in nematic phase of the LC for $T-T_{\mathrm{NI}}=-2.8 \mathrm{~K}$ and $\gamma_{1}=20 \mathrm{~N} / \mathrm{m}^{2}<\gamma_{1}^{(c)}$. Thick lines indicates phase boundary, while the representative thin lines connects the coexisting pairs $\left(\Phi_{2 p}, \Phi_{2 n}\right)$ and $\left(\Phi_{3 p}, \Phi_{3 n}\right)$. For the explication of the meaning of regions I, II, and III see the main text. The letters $\mathrm{N}$ and $\mathrm{P}$ denote nematic and paranematic ordering of CNTs, respectively.

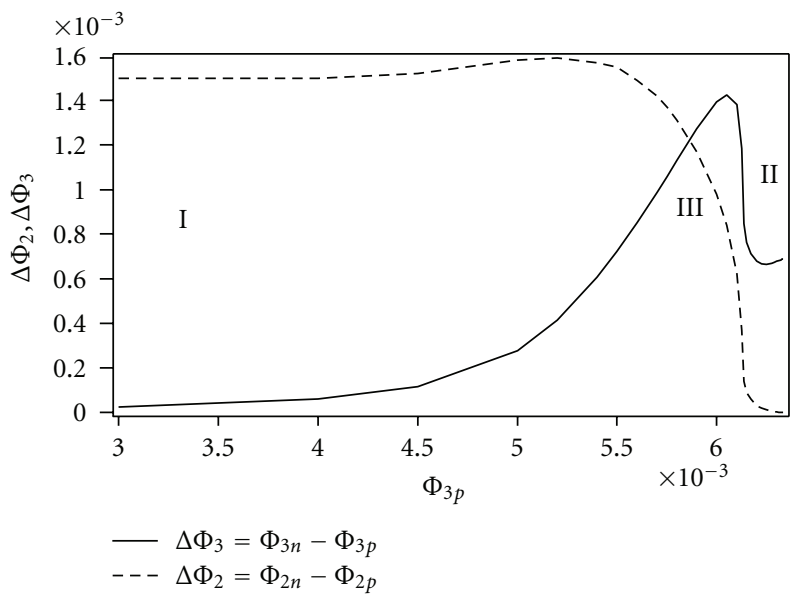

Figure 3: The difference of volume fraction of both CNTs types dispersed in nematic phase of LC for $T-T_{\mathrm{NI}}=-2.8 \mathrm{~K}$ and $\gamma_{1}=$ $20 \mathrm{~N} / \mathrm{m}^{2}<\gamma_{1}^{(c)}$. For the meaning of regions I, II, and III see the main text.

containing only one types of CNTs of length $L_{03}=800 \mathrm{~nm}$ with a volume fraction gap at the transition $\Delta \Phi_{3}=\Phi_{3 n}-$ $\Phi_{3 p}=0.007022-0.006333=0.000689$ (subregion II). In these two subregions, due to very small values of $\Phi_{3}$ and $\Phi_{2}$, respectively, the coupling term between CNTs types (the $\gamma_{23}$ term in (3)) is relatively small and the types are independent. On the contrary, in the coupled region (region III in Figures 2 and 3), the interaction term in (3) becomes important and the two CNTs types influence each other. In this region, the volume fraction of the longer CNTs increases in the nematic phase, while that of the shorter CNTs decreases.

To see in more details the influence of bidispersity on the order parameters we have plotted in Figure 4 the order parameters profiles for a monodisperse CNTs, while in Figure 5, we have shown the order parameters profiles for the bidisperse system for two fixed values of the volume 


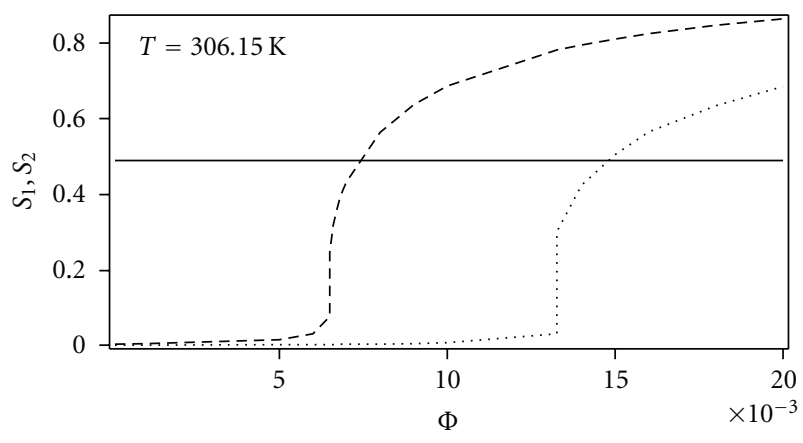

$$
\begin{array}{ll}
\text { - } & S_{1} \\
\ldots . & S_{2}(L=200) \\
--- & S_{2}(L=400)
\end{array}
$$

FIGURE 4: Order parameters profiles for monodisperse CNTs for T$T_{\mathrm{NI}}=-2.8 \mathrm{~K}$ and $\gamma_{1}=20 \mathrm{~N} / \mathrm{m}^{2}<\gamma_{1}^{(c)}$.

fraction of the shorter CNTs. The values of temperatures and coupling parameter are the same as in previous figure: $T-T_{\mathrm{NI}}=-2.8 \mathrm{~K}$ and $\gamma_{1}=20 \mathrm{~N} / \mathrm{m}^{2}<\gamma_{1}^{(c)}$.

Figure 4 shows that in the case of a monodisperse system below the critical point $\left(\gamma_{1}<\gamma_{1}^{(c)}\right)$ the paranematic-nematic phase transition is first order (discontinuity in the order parameter). The transition takes place at a value of volume fraction depending on the ratio $L_{0 i} / D\left(\Phi_{2}^{(\mathrm{NI})}=0.0133\right.$ for $L_{2}=200$ and $\Phi_{3}^{(\mathrm{NI})}=0.0065$ for $\left.L_{3}=400\right)$. Longer CNTs exhibit nematic ordering at a lower volume fraction. In the nematic phase, the longer CNTs are more ordered than the shorter ones. Note that a value of the order parameter of the LC is almost constant on varying $\Phi$ if Figure 4 (i.e., value of $S_{1}$ is dominated by temperature, not by the volume fraction of CNTs). Due to much larger free energy of the LC (there are many more molecules of LC per unit volume than there are CNTs) the CNTs types are enslaved by LC (the LC fluid dictates the behavior of CNTs).

The order parameters profiles in Figure 5(a) are represented for a value of $\Phi_{2}$ less than the corresponding $\Phi_{2}^{(\mathrm{NI})}$ for the monodisperse system with $L_{2}=200$. However, the increasing volume fraction of longer CNTs induces a very weakly first-order paranematic-nematic phase transition of shorter CNTs, with a very small jump in the order parameter. This transition takes place at the same value $\Phi_{3}$ as the stronger paranematic-nematic first-order phase transition of longer CNTs (it is a hidden paranematic-nematic first-order phase transition which takes place inside the coexistence region in Figure 3). In the nematic phase, the longer CNTs are more ordered than the shorter ones. In Figure 5(b), we have shown the order parameter profiles for a value of $\Phi_{2}$ very close to $\Phi_{\mathrm{NI}}$ for themonodisperse system with $L_{2}=$ 200. Now, the jump of order parameter of the shorter CNTs becomes larger that the corresponding quantity for longer CNTs, but in the nematic phase the order in longer CNTs remains larger than that of the shorter CNTs.

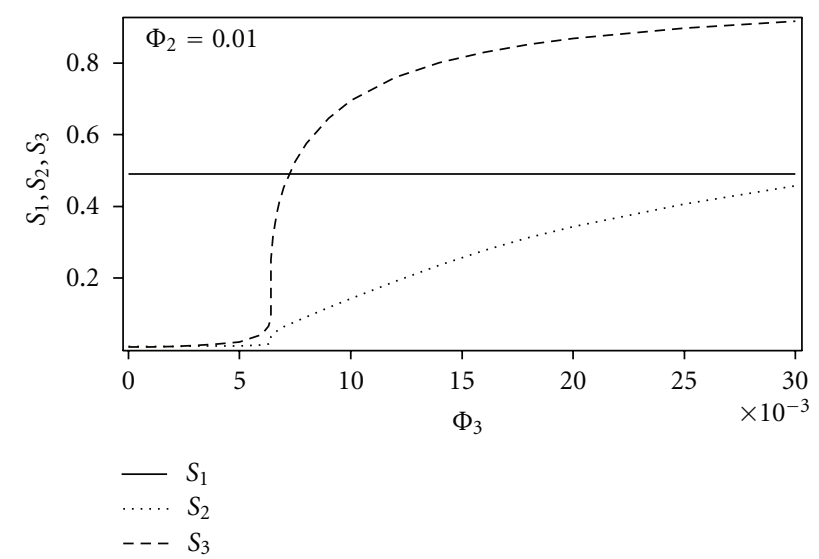

(a)

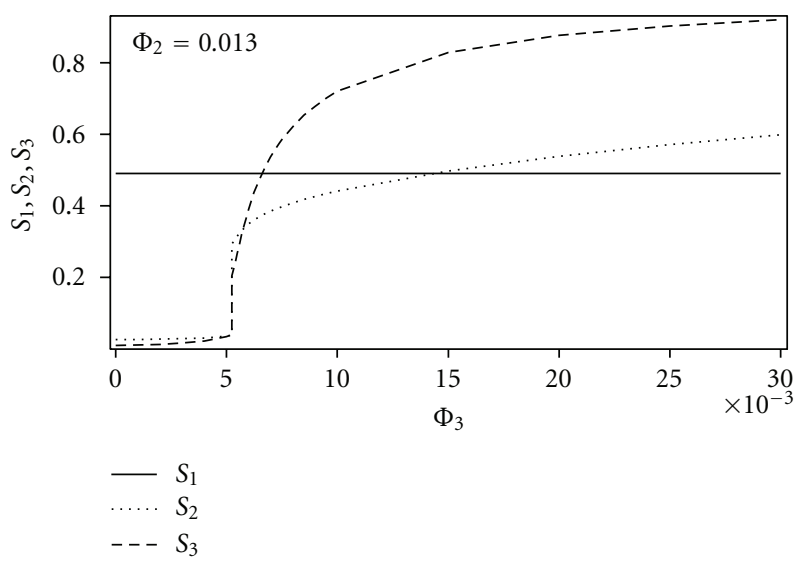

(b)

FIGURE 5: Order parameters profiles for two fixed values of the volume fractions of shorter CNTs for $T-T_{\mathrm{NI}}=-2.8 \mathrm{~K}, \gamma_{1}=$ $20 \mathrm{~N} / \mathrm{m}^{2}<\gamma_{1}^{(c)}$.

3.4. Supercritical Regime. In the case of a more realistic value of the coupling parameter between the LC molecules and CNTs $\gamma_{1}=10^{3} \mathrm{~N} / \mathrm{m}^{2}>\gamma_{1}^{(c)}$, we plot in Figure 6 the order parameter profiles for a monodisperse system. In Figure 7, we plot order parameter profiles for the bidisperse system for a fixed value of the volume fraction of shorter CNTs at the same temperature $T-T_{\mathrm{NI}}=-2.8 \mathrm{~K}$.

Figure 6 shows that in the case of monodisperse system above a critical point $\left(\gamma_{1}>\gamma_{1}^{(c)}\right)$, the alignment of the CNTs gradually increases on increasing their volume fraction. The longer CNTs are more ordered than the shorter ones and the CNTs types are enslaved by LC (the order parameter of LC depends only on temperature, not on the volume fractions of CNTs).

The degree of ordering $S_{i}$ in bidispersed system is demonstrated in Figure 7. We display $S_{i}=S_{i}\left(\Phi_{3}\right)$ dependencies at constant $\Phi_{2}$. The order parameter of the shorter CNTs increases but not significantly with the volume fraction of the longer CNTs (Figure 7). In relatively well-oriented state, the orientational ordering of longer CNTs is larger than that of a shorter rods. 


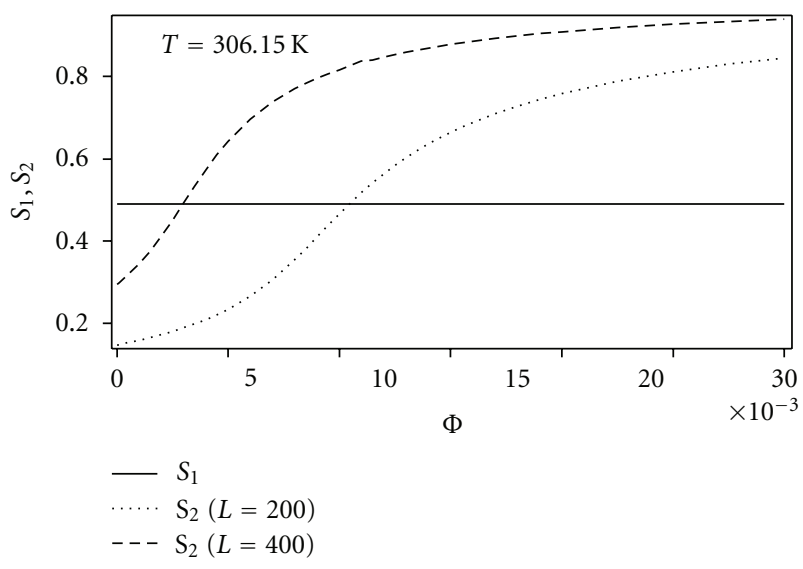

FIGURE 6: Order parameters profiles for monodisperse CNTs for T$T_{\mathrm{NI}}=-2.8 \mathrm{~K}, \gamma_{1}=10^{3} \mathrm{~N} / \mathrm{m}^{2}>\gamma_{1}^{(c)}$.

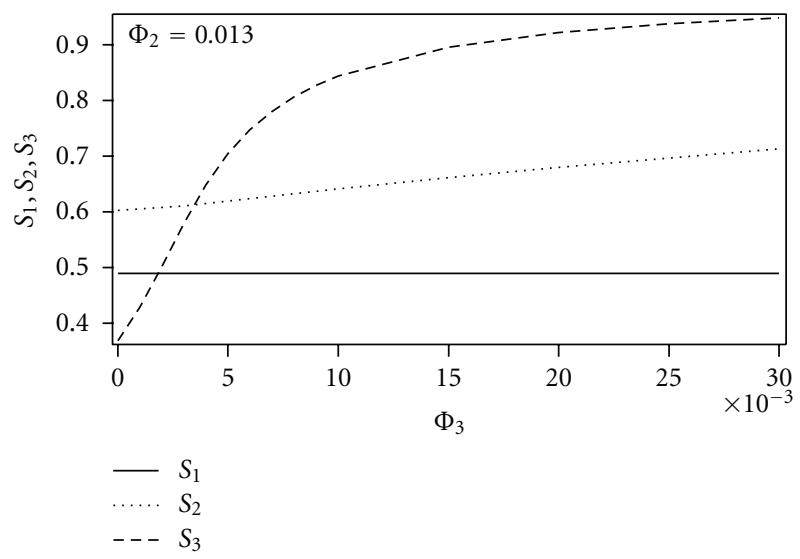

Figure 7: Order parameters profiles for a fixed value of the volume fraction of shorter CNTs for $T-T_{\mathrm{NI}}=-2.8 \mathrm{~K}, \gamma_{1}=10^{3} \mathrm{~N} / \mathrm{m}^{2}>\gamma_{1}^{(c)}$.

\section{Conclusions}

In the present paper, we extend the mesoscopic model $[28,29]$ to include length bidispersity of CNTs dispersed in LC in the weak anchoring limit of the coupling between LC molecules and CNTs (in this limit, the coupling is dominated by the anisotropy of the surface tension not by the deformation of the director field). The main conclusions of our study can be summarized as follows.

(1) Depending on the coupling between the LC molecules and CNTs (the value of the coupling constant $\gamma_{1}$ ), two different regimes can be defined: (i) if $\gamma_{1}<$ $\gamma_{1}^{(c)}$, the nematic-isotropic phase transition of CNTs dispersed in LC is first order and (ii) $\gamma_{1}>\gamma_{1}^{(c)}$ corresponds to a supercritical transformation (the evolution of the order parameter is gradual). $\gamma_{1}^{(c)}$ is the critical value of the coupling parameter depending on the temperature (Figure 1). In both regimes, the isotropic phase of CNTs transforms into a phase with a small degree of ordering, a paranematic phase.
Above the critical point, this degree of orientational order is strongly increasing.

(2) The CNTs are enslaved by the LC (the nematic LC order parameter depends only on the temperature not on the volume fractions of the CNTs).

(3) The longer CNTs are driven into the nematic phase $\left(\Phi_{3 n}-\Phi_{3 p}>0\right.$ in Figures 2 and 3$)$.

(4) In region III from Figures 2 and 3, the longer CNTs induce a larger differences in the volume fraction $\Phi_{2 n}-\Phi_{2 p}>0$ for the shorter CNTs.

(5) In the nematic phase, the longer CNTs are more ordered than the shorter ones $\left(S_{3 n}-S_{2 n}>0\right.$ in Figures 5 and 7).

Finally, we point out the mesoscopic model for length bidisperse CNTs dispersed in LC presented here is only a first step in considering the important influence of polydispersity on the ordering of CNTs in nematic fluids. Together with considering the attraction interaction between CNTs, this subject will be studied in a future work.

\section{Acknowledgments}

V. Popa-Nita gratefully acknowledges the hospitality and the supporting grant of Faculty of Natural Sciences and Mathematics, University of Maribor, Slovenia. Another grant from the Romanian National Authority for Scientific Research, CNCSIS-UEFISCDI, Project no. PN-II-ID-PCE2011-3-1007 is also acknowledged.

\section{References}

[1] R. Saito, M. S. Dresselhouse, and G. Dresselhouse, Physical Properties of Carbon Nanotubes, Imperial Gollege Press, London, UK, 1998.

[2] C. Zakri, "Carbon nanotubes and liquid crystalline phases," Liquid Crystals Today, vol. 16, no. 1, pp. 1-11, 2007.

[3] S. Zhang and S. Kumar, "Carbon nanotubes as liquid crystals," Small, vol. 4, no. 9, pp. 1270-1283, 2008.

[4] J. P. F. Lagerwall and G. Scalia, "Carbon nanotubes in liquid crystals," Journal of Materials Chemistry, vol. 18, pp. 28902898, 2008.

[5] M. Rahman and W. Lee, "Scientific duo of carbon nanotubes and nematic liquid crystals," Journal of Physics D, vol. 42, Article ID 063001, 2009.

[6] M. D. Lynch and D. L. Patrick, "Organizing carbon nanotubes with liquid crystals," Nano Letters, vol. 2, no. 11, pp. 11971201, 2002.

[7] I. Dierking, G. Scalia, and P. Morales, "Liquid crystal-carbon nanotube dispersions," Journal of Applied Physics, vol. 97, Article ID 044309, 5 pages, 2005.

[8] J. Lagerwall, G. Scalia, M. Haluska, U. Dettlaff-Weglikowska, S. Roth, and F. Giesselmann, "Nanotube alignment using lyotropic liquid crystals," Advanced Materials, vol. 19, no. 3, pp. 359-364, 2007.

[9] N. Lebovka, T. Dadakova, L. Lysetskiy et al., "Phase transitions, intermolecular interactions and electrical conductivity behavior in carbon multiwalled nanotubes/nematic liquid crystal composites," Journal of Molecular Structure, vol. 887, no. 1-3, pp. 135-143, 2008. 
[10] F. Brochard and P. G. de Gennes, "Theory of magnetic suspensions in liquid crystals," Journal of Physics, vol. 31, pp. 691-708, 1970.

[11] S. V. Burylov and Y. L. Raikher, "On the orientation of an anisometric particle suspended in a bulk uniform nematic," Physics Letters A, vol. 149, no. 5-6, pp. 279-283, 1990.

[12] S. V. Burylov and Y. L. Raikher, "Orientation of a solid particle embedded in a monodomain nematic liquid crystal," Physical Review E, vol. 50, no. 1, pp. 358-367, 1994.

[13] D. Andrienko, M. P. Allen, G. Skačej, and S. Žumer, "Defect structures and torque on an elongated colloidal particle immersed in a liquid crystal host," Physical Review E, vol. 65, no. 4, Article ID 041702, 7 pages, 2002.

[14] D. Andrienko, M. Tasinkevych, P. Patricoi, and M. M. Telo da Gamma, "Forces between elongated particles in a nematic colloid," Physical Review E, vol. 68, Article ID 051702, 2003.

[15] D. Andrienko, M. Tasinkevych, P. Patricoi, and M. M. Telo da Gamma, "Interaction of colloids with a nematic-isotropic interface," Physical Review E, vol. 69, Article ID 021706, 2004.

[16] D. Andrienko, M. Tasinkevych, and S. Dietrich, "Effective pair interactions between colloidal particles at a nematic-isotropic interface," Europhysics Letters, vol. 70, pp. 95-101, 2005.

[17] F. R. Hung, O. Guzman, B. T. Gettelfinger, N. L. Abbott, and J. J. de Pablo, "Anisotropic nanoparticles immersed in a nematic liquid crystal: defect structures and potentials of mean force," Physical Review E, vol. 74, Article ID 011711, 2006.

[18] M. J. Green, N. Behabtu, M. Pasquali, and W. W. Adams, "Nanotubes as polymers," Polymer, vol. 50, no. 21, pp. 49794997, 2009.

[19] Y. Sabba and E. L. Thomas, "High-concentration dispersion of single-wall carbon nanotubes," Macromolecules, vol. 37, no. 13, pp. 4815-4820, 2004.

[20] A. M. Somoza, C. Sagui, and C. Roland, "Liquid-crystal phases of capped carbon nanotubes," Physical Review B, vol. 63, Article ID 081403-1, 2001.

[21] M. J. Green, A. N. G. Parra-Vasquez, N. Behabtu, and M. Pasquali, "Modeling the phase behavior of polydisperse rigid rods with attractive interactions with applications to singlewalled carbon nanotubes in superacids," Journal of Chemical Physics, vol. 131, Article ID 084901, 2009.

[22] Z. Kutnjak, S. Kralj, and S. Žumer, "Effect of dispersed silica particles on the smectic-A-smectic-C* phase transition," Physical Review E, vol. 66, no. 4, Article ID 041702, 8 pages, 2002.

[23] G. Cordoyiannis, G. Nounesis, V. Bobnar, S. Kralj, and Z. Kutnjak, "Confinement-induced orientational order in a ferroelectric liquid crystal containing dispersed aerosils," Physical Review Letters, vol. 94, no. 2, Article ID 027801, 2005.

[24] E. Karatairi, B. Rožič, Z. Kutnjak et al., "Nanoparticle-induced widening of the temperature range of liquid-crystalline blue phases," Physical Review E, vol. 81, no. 4, Article ID 041703, 2010.

[25] G. Cordoyiannis, P. Losada-Pérez, C. S. P. Tripathi et al., "Blue phase III widening in CE6-dispersed surface-functionalised CdSe nanoparticles," Liquid Crystals, vol. 37, no. 11, pp. 14191426, 2010.

[26] S. Kralj, R. Rosso, and E. G. Virga, "Finite-size effects on order reconstruction around nematic defects," Physical Review E, vol. 81, Article ID 021702, 2010.

[27] M. Cvetko, M. Ambrožič, and S. Kralj, "Memory effects in randomly perturbed systems exhibiting continuous symmetry breaking," Liquid Crystals, vol. 36, no. 1, pp. 33-41, 2009.
[28] P. Van der Schoot, V. Popa-Nita, and S. Kralj, "Alignment of carbon nanotubes in nematic liquid crystals," Journal of Physical Chemistry B, vol. 112, no. 15, pp. 4512-4518, 2008.

[29] V. Popa-Nita and S. Kralj, "Liquid crystal-carbon nanotubes mixtures," Journal of Chemical Physics, vol. 132, no. 2, Article ID 024902, 2010.

[30] W. Song and A. H. Windle, "Isotropic-nematic phase transition of dispersions of multiwall carbon nanotubes," Macromolecules, vol. 38, no. 14, pp. 6181-6188, 2005.

[31] P. G. de Gennes and J. Prost, The Physics of Liquid Crystals, Oxford University Press, Oxford, UK, 1993.

[32] P. J. Flory, "Phase equilibria in solutions of rod-like particles," Proceedings of the Royal Society A, vol. 243, pp. 73-89, 1956.

[33] L. Onsager, "The effects of shape on the interaction of colloidal particles," Annals of the New York Academy of Sciences, vol. 51, pp. 627-659, 1949.

[34] M. Doi, Journal of Polymer Science Part B, vol. 19, p. 229, 1981.

[35] N. Kuzuu and M. Doi, Journal of the Physical Society ofJapan, vol. 52, p. 3486, 1983.

[36] M. Doi and S. F. Edwards, Theory of Polymer Dynamics, Clarendon, Oxford, UK, 1989.

[37] V. Popa-Nita, "unpublishedresults".

[38] P. Oswald and P. Pieranski, Nematic and Cholesteric Liquid Crystals, Concepts and Physical Properties Illustrated by Experiments, Liquid Crystals Book Series, Taylor and Francis Group, CRC Press, Boca Raton, Fla, USA, 2005.

[39] S. Faeti and V. Palleshi, "Measurements of the interfacial tension between nematic and isotropic phase of some cyanobiphenyls," Journal of Chemical Physics, vol. 81, Article ID 6254, 1984.

[40] S. Faeti and V. Palleshi, "Nematic-isotropic interface of some members of the homologous series of 4-cyano- 4 '-(nalkyl)biphenyl liquid crystals," Physical Review A, vol. 30, pp. 3241-3251, 1984.

[41] S. Faeti and V. Palleshi, "Molecular orientation and anchoring energy at the nematic-isotropic interface of7CB," Journal de Physique Lettres, vol. 45, pp. 313-318, 1984. 

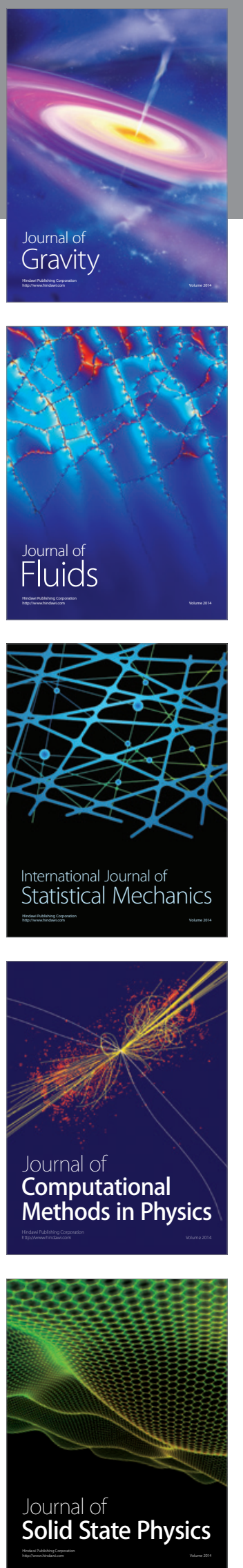

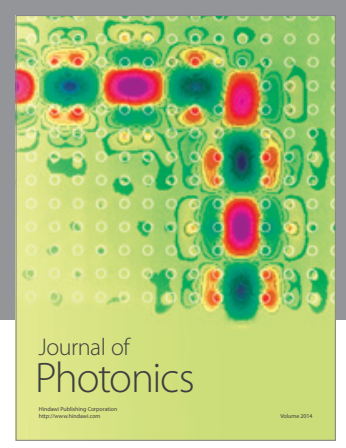

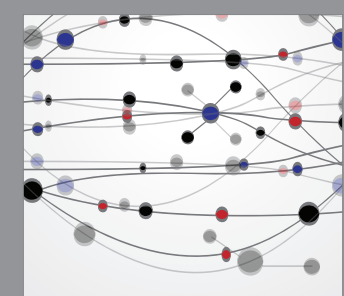

The Scientific World Journal
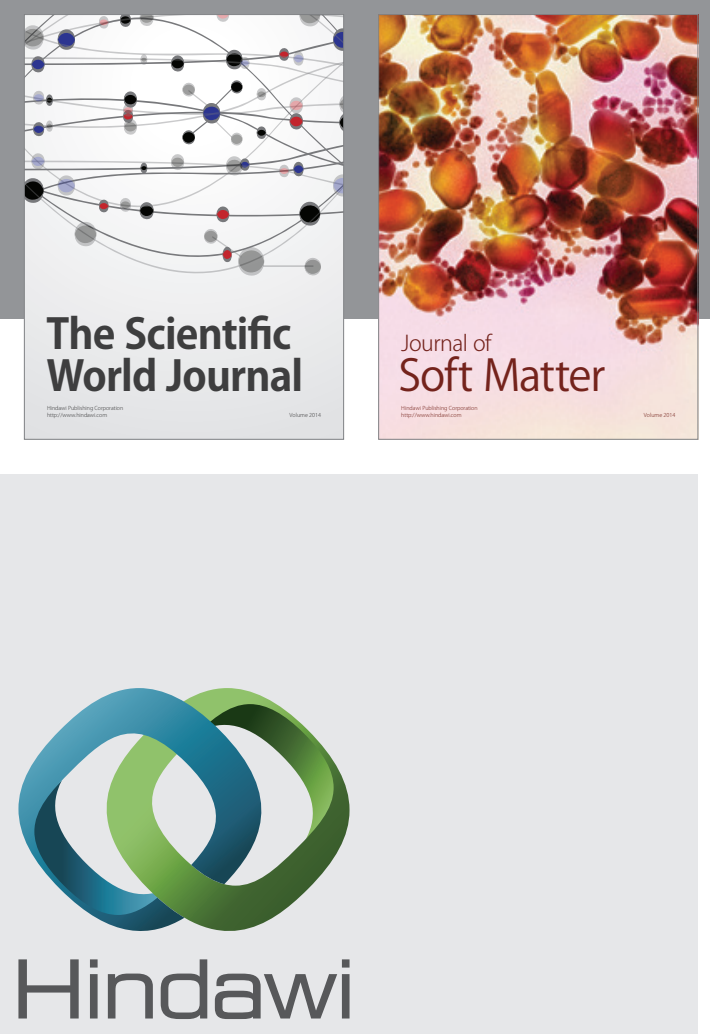

Submit your manuscripts at

http://www.hindawi.com
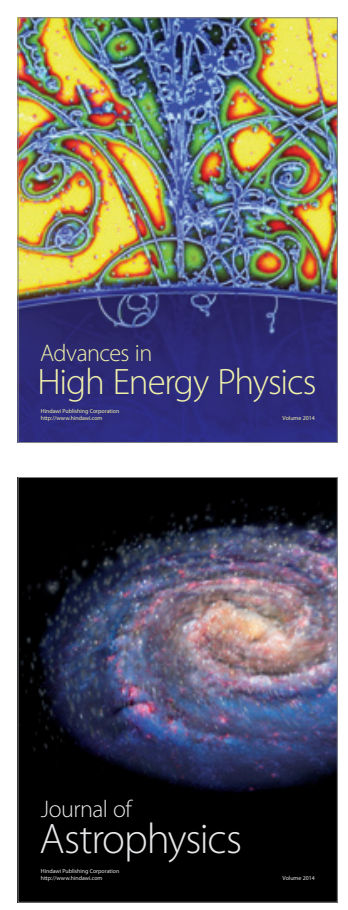
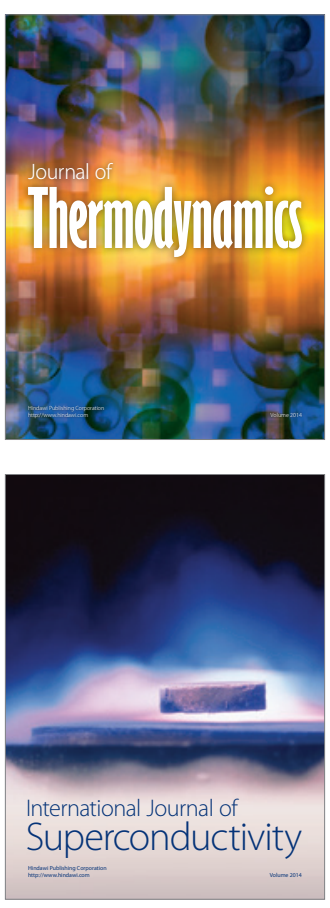
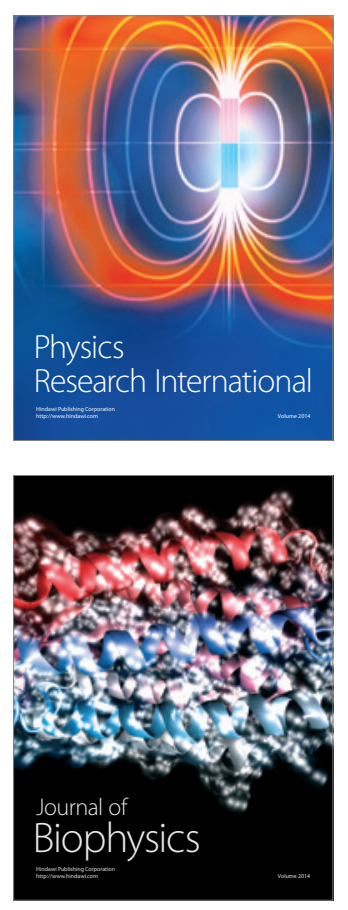
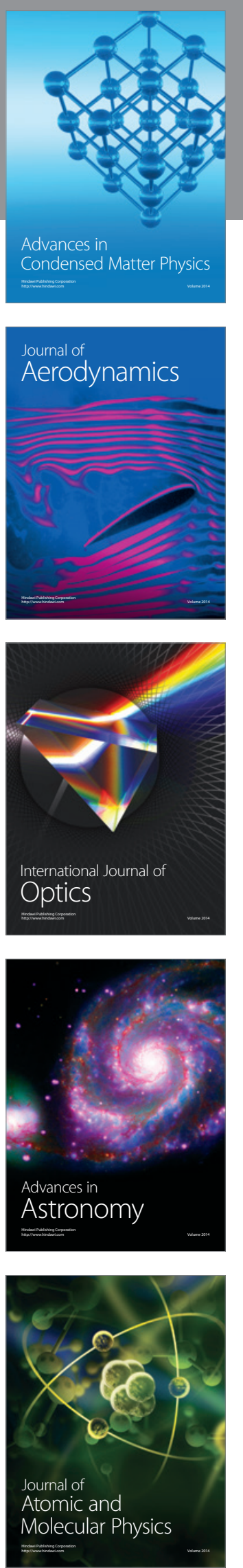